\title{
SENESCENCE IN VITRO AND IONISING RADIATIONS-THE HUMAN DIPLOID FIBROBLAST MODEL
}

\author{
A. F. G. STEVENSON* \\ Institut für Anatomie, Universität Regensburg, D-8400 Regensburg (F.R.G.) \\ T. CREMER \\ Institut für Anthropologie und Humangenetik, Universität Heidelberg, D-6900 Heidelberg (F.R.G.) \\ (Received September 18, 1979; in revised form August 4, 1980)
}

\section{SUMMARY}

The influence of ionising radiations on ageing is still controversial. Since Hayflick established the concept that diploid cells have finite lifespan in vitro, human diploid fibroblast (HDF) cultures have been recognised as a potent experimental model for cytogerontological investigations. In this study HDF cultures in phase II were exposed to acute irradiation with either $\mathrm{X}$-rays or fast neutrons. The replicative potentials and labelling indices with $\left[{ }^{3} \mathrm{H}\right]$ thymidine were measured post irradiation until the cultures ceased growth in phase III. Cell mortality was measured by cloning. The apparent loss in replicative potential of irradiated mass cultures was wholly attributable to the loss of viable clonogenic cells. The current concept of precocious clonal senescence in vitro as a late effect of irradiation in clonogenic survivors is not supported by the present experiments. Instead, our results suggest that exposure to a single dose of ionising radiations either causes total replicative incapacitation (killing) of HDF cells and their progeny early after irradiation or leaves their replicative potentials unperturbed.

\section{INTRODUCTION}

Embryonic fibroblasts are easily established in vitro. Such euploid cells of human and avian origin are known to be remarkably stable in culture [1-4]; spontaneous transformation is unknown and radiation-induced transformation is rarely ever observed. Although Weismann $[5,6]$ had already put forward a theory in 1881 that evolution led to a finite replicative lifespan of somatic tissue cells in animals but not necessarily in plants, the early experiments of Carrel [7] and Ebeling [8] expounded the concept that cells had unlimited lifespans outside the organism. Deterioration of a culture was wholly attributed to inadequacies of culture media. This notion of cellular immortality was

*Address correspondence to: Dr. A. F. G. Stevenson, Institut für Anatomie, Universität Regensburg, Universitätsstrasse 31, D-8400 Regensburg, Federal Republic of Germany. 
generally accepted until Hayflick and Moorhead [9] brought forward evidence that challenged this dogma. Hayflick $[9,10]$ introduced the concept of in vitro senescence which contends that diploid fibroblasts in culture have a limited replicative potential. The decline in growth (Hayflick's phase III) is deemed an intrinsic manifestation of cellular ageing, terminating in cessation of growth. While the disability of fibroblast cultures to make infinite population doublings is recognised, the interpretation of this phenomenon remains controversial. A current theory indeed attributes unlimited replicative potentials to "uncommitted" cells in human diploid fibroblast (HDF) cultures [11-13].

HDF cultures do, none the less, serve as a convenient in vitro model for cytogerontological investigations. Two main groups of theories which propose (1) that cellular ageing is due to random accumulation of errors in information handling macromolecules $[14,15]$ or $(2)$ that ageing depends on a set of programmed events [16], have provoked zealous efforts for verification. HDF cultures have been employed for such experimental studies [17-20]. In view of these theories, it has been of interest to know if agents such as ionising radiations could possibly alter the process leading to senescence. The early pioneering work of Russ and Scott [21] and several later studies (for review see ref. 22) indicate that irradiation of laboratory rodents induced shortening of their lifespans. These studied meet with difficulties in analysis of causative factors [22]. Substitution of animals by HDF cultures offers, on the other hand, a more easily controlled system.

When the growth densities of HDF cultures maintained in serial cultivation are plotted as a function of the population doubling level (PDL), such growth curves in phase III typically take the form of a shouldered curve, reminiscent of radiation dose-effect curves. This has prompted cytogerontologists to speculate on an analogous target-hit theory [23] of cellular inactivation through either multiple hits or multiple targets, after a critical threshold has been reached [10]. The putative parallelism in the mode of action argues in favor of the use of ionising radiation in experimental attempts at influencing the course of events leading to cellular senescence. A few studies have been reported by Maciera-Coelho et al. [1, 2, 4, 24-26], Laublin et al. [27] and Ban et al [28]. They suggest that irradiation of human [4, 26-28] as well as chicken [24] diploid fibroblast cultures can induce precocious senescence. The same effect was also reported for the clonal lifespan of Paramecia [29]. These different studies are in keeping with the view (generated from observations on irradiated animals) that ionising radiations accelerate natural ageing.

A critical re-valuation of animal studies $[22,30]$ and of epidemiological data from Hiroshima and Nagasaki [31] has cast strong doubt on the ascribed ageing effects of ionising radiations. With regard to the emerging view that irradiation does not influence the process of ageing, we have used the HDF model to gain data from our own, using another diploid fibroblast cell line. Our experimental protocols differed from those of reported studies. Our estimations of the population doubling capacity of quiescent phase II cultures after exposure to either X-rays or fast neutrons suggest that the earlier termination of irradiated cultures was wholly attributable to the loss of clonogenic cells. This result negates the concept that clonogenic survivors of irradiation would manifest a reduced replicative lifespan and stands in contrast to findings of other investigators. This incongruency is discussed. 


\section{MATERIALS AND METHODS}

\section{Human diploid fibroblasts}

A commercially available HDF cell strain, which was derived from lung tissue of a 14-15 week-old male negro fetus, was obtained from Flow Laboratories at PDL 18 (Flow 2000, code 0-13200). Chromosome analysis with Q-banding (courtesy of D. Hager and Dr. U. Müller, Institute of Anthropology and Human Genetics, University of Heidelberg) revealed a normal male human karyotype. The scheme of subcultivation at confluency followed the 1:2 split ratio up to PDL 28 . Such early phase II cells were cryopreserved in liquid nitrogen, and served as starting material for experimental work.

\section{Culture conditions}

The cells were grown in Eagle's MEM having Earle's salts, with $12 \%$ fetal calf serum (FCS), non-essential amino acids, penicillin (100 I.U./ml), streptomycin $(100 \mu \mathrm{g} / \mathrm{ml})$ and $2.2 \mathrm{~g} / 1 \mathrm{NaHCO}_{3}$. Medium was freshly prepared and adjusted to $\mathrm{pH} 7.4$ before use. Cultures were incubated at $37{ }^{\circ} \mathrm{C}$ with $5 \% \mathrm{CO}_{2}$ and saturated humidity. Growth medium $(0.3$ $\mathrm{ml} / \mathrm{cm}^{2}$ of growth area) was replaced with fresh medium twice weekly in all serial cultures. Periodic screening for Mycoplasma contamination was carried out by the method of double-labelling with $\left[{ }^{3} \mathrm{H}\right]$ uracil and $\left[{ }^{14} \mathrm{C}\right]$ uridine [32]. Uridine/uracil ratios of over 400 were consistently obtained. The cultures were therefore regarded as non-contaminated.

Cultures were detached and dispersed at $37{ }^{\circ} \mathrm{C}$ in a modified Hanks' balanced salt solution (HBSS), free of calcium and magnesium ions, containing $0.25 \%$ (w/v) Trypsin (Serva, $1: 250$ ) and $0.02 \%$ disodium EDTA. All solutions were sterilised by filtration (0.22 $\mu \mathrm{m}$ Millipore).

\section{Estimation of population doubling level (PDL)}

Cells were counted using a haemocytometer, diluted accordingly and sown into plastic culture flasks (Falcon Plastics) with $75 \mathrm{~cm}^{2}$ growth area. The initial density for vigorously growing cultures was generally about $8 \times 10^{3}$ cells $/ \mathrm{cm}^{2}$ growth surface. Phase II cultures attained confluency after one week [33]. The experimental designs in different series consisted basically of subcultivation of serial cultures either at confluency or during logarithmic growth. When it was felt that growth rates were slowing down (approach to phase III), more time was correspondingly allowed before subculture. In senescing cultures, the initial plating was also increased to facilitate growth. The extent of population doubling (PD) made by a culture was calculated from the initial and final number of cells obtained at each subcultivation. The formula $\log _{2} n / n_{0}=$ PD was employed, where $n_{0}=$ initial number and $n=$ final number of cells. Addition of PDs yielded the PDL or cumulative population doublings (CPD). The lifespan of HDF-strain Flow 2000 in our hands ranged from 68 to 76 CPDs.

\section{Cloning of $H D F$}

The fraction of cells surviving radiation treatment was estimated by cloning experimental cells with feeder cells derived from the same culture [34]. Feeder cells were X- 
irradiated with $4 \mathrm{kR}$ as attached confluent cultures before trypsinisation and plating in Petri dishes. The number of experimental cells was increased with radiation dose, in order to obtain a reasonable number of countable colonies. The density of cells plated out (experimental cells plus feeder cells) was kept at $2 \times 10^{3}$ cells $/ \mathrm{cm}^{2}$ growth surface. The medium employed for cloning experiments was the same as for serial cultures, except that FCS was $20 \%$. Partial replacement of medium (50\%) was done twice weekly. This gave a plating efficiency of about 15-25\%, which was considered satisfactory. An aggregate of 50 or more cells was deemed a clone. After 14 days incubation under the same conditions, clones generally consisted of $10^{3}$ to $3 \times 10^{3}$ cells. The clones were fixed in Carnoy $\mathrm{A}$ solution and stained with Giemsa before scoring under a dissection microscope.

\section{Irradiation of HDF cultures}

Attached confluent cultures were given a single exposure to ionising radiations under cover of culture medium. X-irradiation was delivered from a Müller type MG 300 machine, operated at $250 \mathrm{kV}$ and $12 \mathrm{~mA}$. The half-value layer was $1.55 \mathrm{~mm} \mathrm{Cu}$. The exposure rate was $93 \mathrm{R} / \mathrm{min}$. In one experiment the exposure rate was reduced to $8 \mathrm{R} / \mathrm{min}$. A duplex dosimeter was used.

Similar cultures were also irradiated with predominantly monoenergetic fast neutrons which had a mean energy of $15 \mathrm{MeV}$. Fast neutrons were produced from a 'van de Graaf generator', using the D-T reaction. The exposure rate for neutron irradiation was 6-8 $\mathrm{rad} / \mathrm{min}$. The measurement of neutron flux densities was through the induced activa. tion of aluminium foils.

\section{Autoradiography and labelling index}

The percentage of cells at each serial subcultivation capable of DNA synthesis was measured by exposing cultures plated at a density of $8 \times 10^{3} \mathrm{cells} / \mathrm{cm}^{2}$ to $\left[{ }^{3} \mathrm{H}\right]$ thymidine. The cultures had a final $\left[{ }^{3} \mathrm{H}\right]$ thymidine concentration of $0.05 \mu \mathrm{Ci} / \mathrm{ml}$ (specific activity $=$ $5 \mathrm{Cu} / \mathrm{mmole}$ ) and were incubated for 72 hours before they were washed with HBSS and fixed in Carnoy A solution. Cristofalo's labelling index [35] was determined microscopically after cultures had been autoradiographed, using Kodak NTB-2 nuclear emulsion, and then Giemsa-stained. About 500 cells from five randomly selected fields (100 cells/field) were counted from each slide specimen. Each specimen was prepared in duplicate.

\section{RESULTS}

The results shown here graphically are reproducible and represent in part duplicate experiments. With each treatment, cultures were subjected to two schemes of serial subcultivation. In one scheme, subcultivation was at confluency, while in the other it was during the logarithmic growth phase. At each subcultivation, the increase in PDL and the $\left[{ }^{3} \mathrm{H}\right]$ thymidine labelling index were measured. 


\section{Replicative potential after $X$-irradiation}

Figures 1 and 2 demonstrate graphically the replicative capacity of HDF cultures which were given a single acute dose of X-radiation at an exposure rate of $93 \mathrm{R} / \mathrm{min}$. Replicate confluent cultures received $500 \mathrm{R}, 1000 \mathrm{R}$ or $0 \mathrm{R}$ (sham-irradiation controls) and were then serially subcultivated either at confluency or during exponential growth, as shown in Fig. 1 and Fig. 2, respectively. The replicative capacity of the cultures after treatment is indicated in CPDs completed, as a function of time, before termination in phase III. Irradiation reduced the level of population doublings attainable in a dosedependent manner. The reduction of CPDs observed after a radiation burden of $500 \mathrm{R}$ and $1000 \mathrm{R}$ was 3 to 5 and 10 to 13 , respectively.

The fraction of DNA-synthesising cells or Cristofalo's labelling index of such treated cultures in the course of serial subcultivation, is represented in Fig. 3. The fraction of DNA-replicating cells was drastically reduced with dose, immediately after irradiation. Treated cultures then recovered. No differences in the labelling indices between serially subcultivated irradiated and non-irradiated cultures were discernible after recovery and the slopes of the curves on CPD indicated similar growth rates. Furthermore, we obtained the same saturation densities in irradiated cultures after recovery as in control cultures. The onset of phase III, as marked by the clear fall in the percentage of DNA-synthesising cells, occurred earlier in irradiated cultures. The labelling indices correlated well with the population doubling capacity of the same cultures.

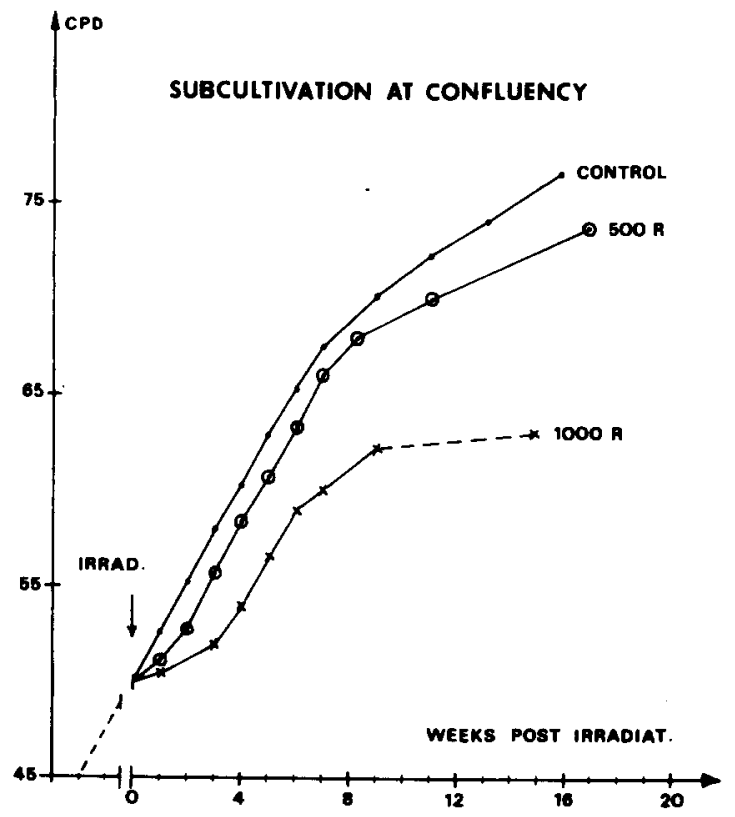

Fig. 1. The replicative potential, measured in terms of the cumulative population doublings (CPD) attained, is shown as a function of time (weeks) post-irradiation. The points on the curves coincide with subcultivation. Cultures were X-irradiated at the PDL indicated and subcultured at confluency. 


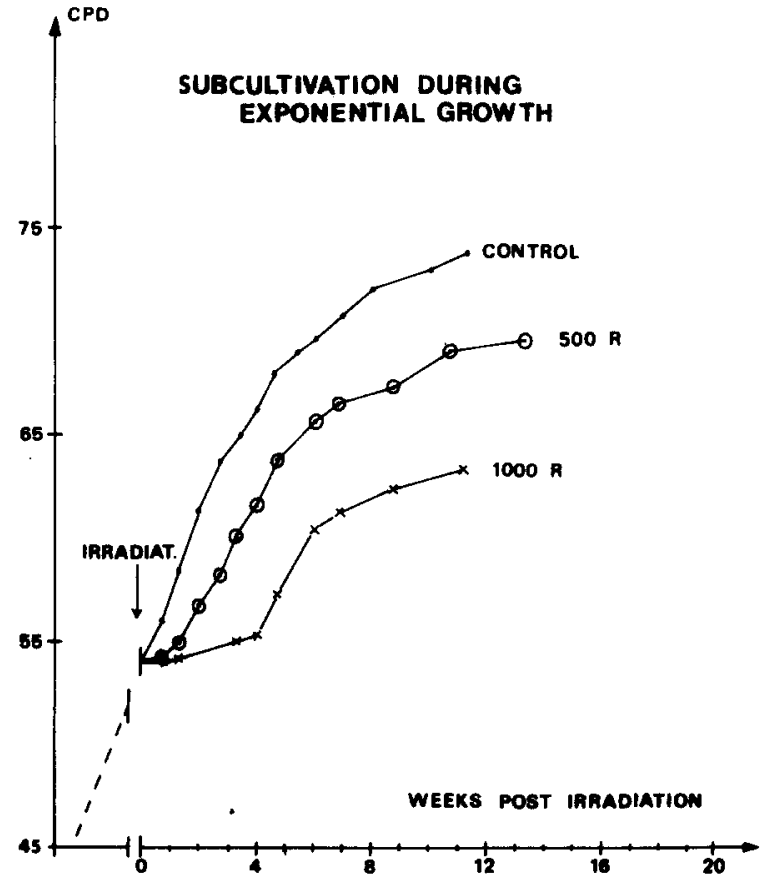

Fig. 2. The replicative potential, measured in terms of the cumulative population doublings (CPD) attained, is shown as a function of time (weeks) post-irradiation. The points on the curves coincide with subcultivation. Cultures were $X$-irradiated at the PDL indicated and subcultured during exponential growth.

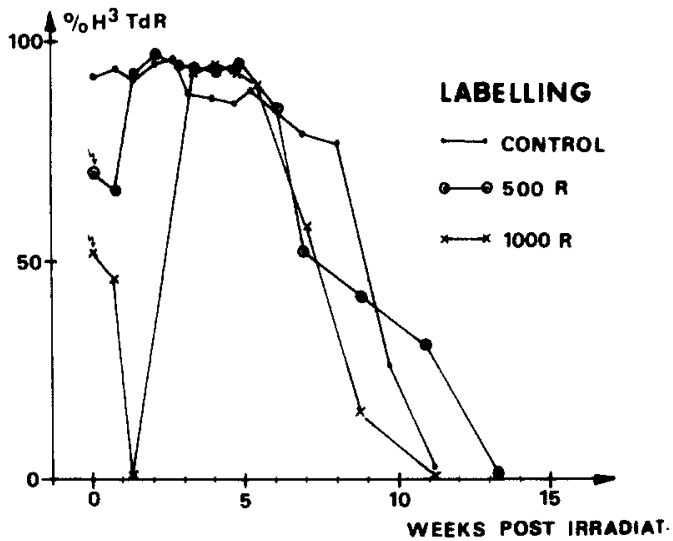

Fig. 3. The percentage of DNA-synthesising cells as evaluated from autoradiograms $\left(\left[{ }^{3} \mathrm{H}\right]\right.$ thymidine labelling) of treated cultures is shown as a function of time (weeks) post-irradiation. After a dosedependent momentary sharp drop, irradiated cultures recovered and were indistinguishable from controls, until a precocious drop in labelling indices was manifested.

\section{Cell mortality after $X$-irradiation}

The sensitivity of HDF to acute X-irradiation at $93 \mathrm{R} / \mathrm{min}$ was estimated in order to afford proper interpretation of data on the replicative capacity of the surviving cell fractions, as demonstrated in Figs. 1 and 2 . A dose-effect curve is shown in Fig. 4. The frac- 


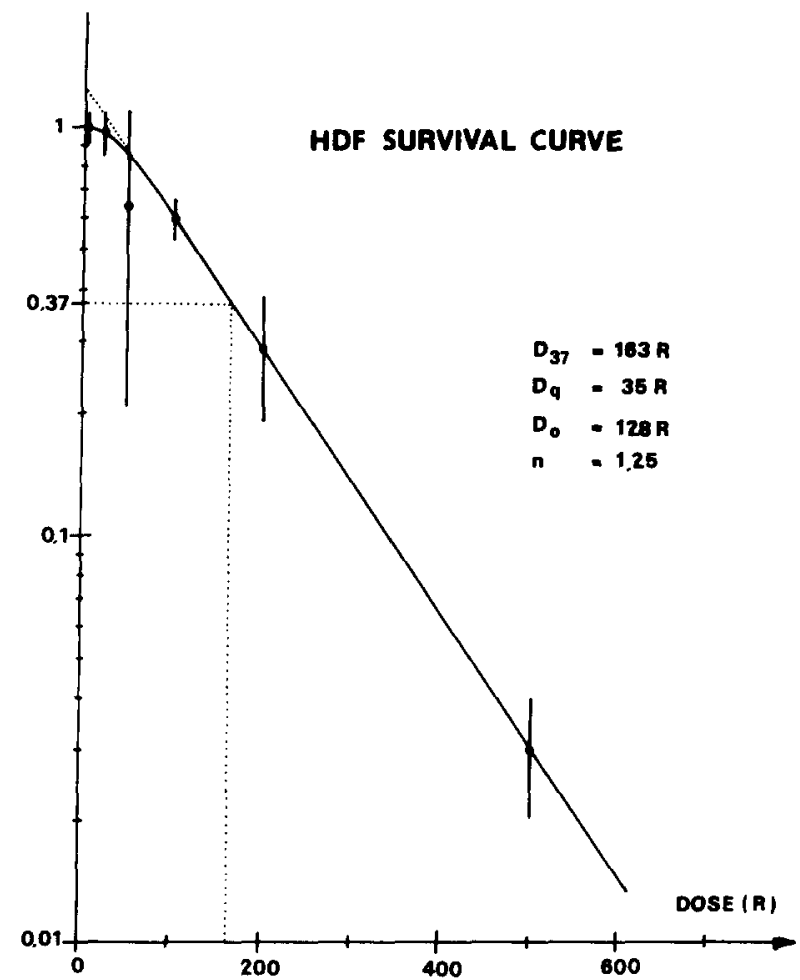

Fig. 4. The mortality curve shows the surviving fraction of clonogenic cells as a function of radiation dose (at $93 \mathrm{R} / \mathrm{min}$ ). The value for $1000 \mathrm{R}$ was estimated by extrapolation of the linearly exponential part of the curve. The radiation sensitivity of this strain of HDF as characterised by $D_{0}$ and extrapolation number $n$, are given on the figure. The vertical bars indicate standard errors.

tion of surviving colony-forming cells (CFC) at radiation doses of $500 \mathrm{R}$ and $1000 \mathrm{R}$ is of interest in these experiments. To estimate this fraction each colony was assumed to be derived from a single fibrocytic cell. The value for cell survival at $1000 \mathrm{R}$ was ascertained by extrapolation of the survival curve. Extrapolation was based on the assumption that the exponential part of the curve remained linear with increasing dose. The CFC survival for $500 \mathrm{R}$, read directly from the survival curve, was about 0.03 . An estimated survival of approximately $0.00018 \mathrm{CFC}$ was obtained for $1000 \mathrm{R}$. The number of additional population doublings which the irradiated cultures had to make in order to reach parity with control cultures (set at $100 \%$ ) may then be calculated from the CFC survival rates, using the above-mentioned formula. Thus, a radiation dose of $500 \mathrm{R}$ (at the given exposure rate) would have required about 5 additional PDs. The surviving fraction of cells was therefore subjected to additional pressure of proliferation.

\section{CPD after fast neutron irradiation}

Fast neutrons are known to have higher radiobiological effectiveness. Monochromatic $15 \mathrm{MeV}$ neutrons were therefore employed for irradiation, in an attempt to see if a qualitatively different change in the replicative behaviour of HDF could be induced. Figure 5 


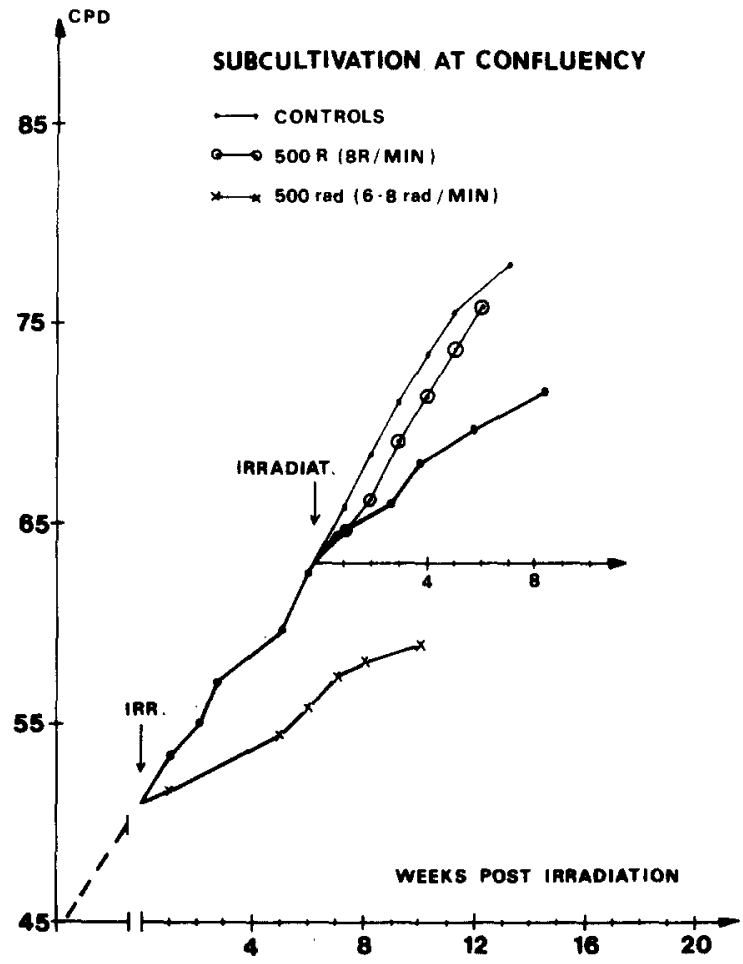

Fig. 5. The replicative potential, measured in terms of the cumulative population doublings (CPD) attained, is shown as a function of time (weeks) post-irradiation. The heavy lines represent the experiment in which fast neutrons $(6-8 \mathrm{rad} / \mathrm{min})$ were employed and corresponds to the outer axis. The inset axis (also time in weeks post irradiation) corresponds to the lighter lines. X-irradiation (lighter lines) at an exposure rate which corresponded to that of neutron irradiation, was at the PDL indicated (late phase II). The ordinate is common to both sets of curves.

represents the results of similar measurements on the replicative capacity of such irradiated cultures. A radiation dose of 500 rad drastically diminished the replicative lifespan (see heavy lines of Fig. 5) by about 13 PDs. The inset axis with the curves in lighter lines indicates the results obtained from a subsequent experiment in which parallel cultures, at the PDL indicated, were irradiated with $500 \mathrm{R}$ X-rays at a dose rate which corresponded with the dose rate at which $15 \mathrm{MeV}$ neutrons were delivered. The results suggest a reduction of 1-2 PD. Cultures for this purpose were obtained from subcultivation of cryopreserved cells of the sham-irradiated control cultures at the PDL indicated. The subcultures originating from the cryo-preserved control material showed a lifespan of approximately 5 CPDs more. In spite of this variation in the replicative lifespans of HDF cultures (see Discussion) a reduction as measured in terms of CPDs before termination of cultures in phase III was consistently observed after irradiation with either X-rays or fast neutrons in all experiments. The replicative lifespan of non-irradiated cultures ranged between 68 and 76 CPDs in seven experiments, while the maximum replicative potential of cultures irradiated with $1000 \mathrm{R}$ of X-rays or $500 \mathrm{rad}$ of fast neutrons was exhausted after $58-64$ CPDs. 


\section{DISCUSSION}

\section{Replicative kinetics in senescence}

The kinetics of cellular senescence in vitro, as measured by the population doubling rate (PDR) and growth densities, has been characterized by some as a gradual decline taking the form of a shouldered slope in phase III $[10,24]$, while others have described precipitous drops [36]. More recent reports have shown that the course of decline in PDR for diploid fibroblast strains cannot be normed [37]. The onset of phase III leading to termination may show anything from a gradual decline to a sharp drop [37]. Our studies with Flow 2000 described phase III as abrupt, leading soon to termination. A range of studies have now established that the replicative potential of HDF strains, expressed in terms of total CPD, may vary widely [20,38,39]. Short-lived and long-lived types are now recognised [38]. We regard Flow 2000 as belonging to the latter category [39].

\section{Radiation inactivation of $H D F$ clonogenicity and replicative potential of clonogenic survivors}

The reduced replicative potential (RP) of irradiated cultures could be attributed to (1) diminution in the number of cells having intact replicative capacities, i.e. loss of cells due to radiation killing, (2) impairment of the replicative potential of clonogenic survivors, and (3) a combination of both cell loss and replicative impairment. In case (1) the dosedependent effects on CPD of irradiated cultures have to be explained by the increased number of cell divisions to which clonogenic survivors were forced in order to repopulate irradiation cultures. In case (2) precocious senescence would indicate that sublethal damage cannot be fully repaired in clonogenic survivors but retains a "memory" of the irradiation insult as proposed by Laublin et al. [27]. Since this latter assumption involves far-reaching consequences with regard to our understanding of late radiation effects in clones from surviving HDF cells and our understanding of ageing in vitro as well, we have carefully considered whether this assumption appears necessary in order to interpret the present data.

The increase in CPDs of irradiated mass cultures, which we have measured during the time of recovery, largely underestimates CPDs of clonogenic survivors for several reasons. After having lost their replicative capacity by ionising radiations, cells were still maintained in a post-replicative state for considerable periods of time as shown by their capability to serve as feeder cells. It is also well-known that cell death may not necessarily occur in parent cells but during several generations after irradiation [40]. In this way cells with potential lethal damage (PLD) which cannot be fully repaired, can be gradually eliminated [40-43]. As an effort to resolve a part of this difficulty of interpretation, the surviving fraction of clonogenic cells was established as a function of radiation dose at the higher exposure rate $(93 \mathrm{R} / \mathrm{min}$ ) (Fig. 4). These data on cell survival after irradiation allowed a rough estimate of the additional proliferative stress of clonogenic survivors during recovery of irradiated cultures as compared with clonogenic cells from shamirradiated cultures. Our calculations yielded values for the earlier exhaustion of the replicative potentials (in terms of CPDs) which coincided with experimental observations. 
The total CPDs observed for cultures irradiated with $500 \mathrm{R}$ and subcultivated at confluency (Fig. 1) was about 1-2 PDs higher than the expected theoretical value. A similar deviation was observed for the labelling indices of the $500 \mathrm{R}$ group. These deviations probably lie within the limits of experimental error and the inherent variability of the system $[13,20,39]$.

After recovery from irradiation the proliferative kinetics of the survivor cells as illustrated by the slopes of the curves on CPD as well as labelling index curves showed no notable differences to those of controls. This may be regarded as further indication that survivor cells able to form macroscopic colonies had completely recovered from the radiation insult. Survivor cells with heritable lesions which might have resulted in reduced growth rates $[40,43]$ were most likely eliminated from the population by our scheme of serial subcultivation. In the light of these considerations any resort to late expression of radiation lesions in clonogenic survivors does not appear to be necessary in order to explain precocious senescence of irradiated HDF cultures in the present experiments. Instead, our data suggest that HDF cultures after recovery from the radiation insult consisted of cells having retained their full replicative potential.

The present results were obtained after irradiation at confluency and do not allow an appraisal of possible effects on cycling cells. Furthermore, in the absence of data on cell survival after irradiation with fast neutrons our conclusion does not necessarily hold for this latter type of irradiation. The more pronounced effect of fast neutrons with high LET (linear energy transfer) was expected as a consequence of the well-known differences in radiobiological characteristics of fast neutrons as compared with X-rays [40].

\section{"Bottle-neck" effect of irradiation}

Even if we were to assume that a decrease in the replicative lifespan of clonogenic survivors was obscured in the present experiments, this would not necessarily indicate a long-term lethal effect of irradiation $[1,4,27]$ in these cells. It has previously been shown that a sudden and drastic reduction of culture size from $2 \times 10^{6}$ cells to $2 \times 10^{3}$ cells, allowing the cultures thereafter to grow back to the normal size, affects the replicative lifespans [13]. Such experiments have been termed "bottle-necks" and a considerable reduction in the number of CPDs before normal death of the cultures was especially observed, when bottle-necks were taken at intermediate passage levels, while bottle-necks early or late in the replicative lifespan showed little effect [13]. Obviously, the effect of irradiation in decimating the pool of potentially replicative cells is comparable to the "bottle-neck" modus and it is noteworthy that no bottle-neck was produced in shamirradiated cultures. Let us assume that the probability of replicative incapacitation by ionising radiation was the same for all cells and that the initial populations of irradiated cells were homogeneous in the sense that they were composed entirely of committed cells, i.e. cells with finite replicative lifespan [13]. Then the commitment theory [11-13] would predict that the fraction of clonogenic survivors was likely composed of cells which produce somewhat reduced average lifespan of irradiated HDF cultures in comparison with sham-irradiated cultures. This result is suggested for the simple reason that cells with lower replicative potentials are more abundant in phase II cultures than cells with 
higher ones. To overcome these difficulties of interpretation it appears necessary to produce similar bottle-necks both in irradiated and sham-irradiated cultures. While these considerations point out that the present experiments did not follow an optimal course in all aspects, they also support our conclusion that it is unnecessary to assume late radiation effects in clonogenic survivors in order to interpret precocious senescence in the present experiments.

\section{Comparison with data from other authors and aspects of future investigations}

Our results stand in contrast to the view expressed by Laublin et al. [27] and Ban et al. [28], who favor the manifestation of radiation late effects in clonogenic survivors as in the form of reduced replicative potentials. The reports that have come from MacieraCoelho $e t$ al. on studies with HDF $[4,26]$ qualify radiation-induced influences as a function of in vitro age. The discrepancies with data and conclusions from other authors cannot be sufficiently explained at the moment. Differences of radiation effects directly attributable to differences in cell strains, radiation quality and radiation schedules (exposure rate, time and number of radiation exposures during the lifespan in vitro) are factors which have to be taken into consideration, but which are exceedingly difficult to assess. For example, Laublin et al. [27] did not find a significant reduction in the lifespan of surviving clones after exposure of HDF cultures to a single dose of 600 rad with ${ }^{137} \mathrm{Cs}$ gamma-rays, which is in agreement with our findings. A significant reduction, however, was noted by these authors when two or three doses of $600 \mathrm{rad}$ were applied at different population doubling levels.

The wide variation in the proliferative potential of clones and subclones in HDF cultures $[13,39]$ poses considerable problems in any attempt to obtain a definite answer to the question of whether or not clonogenic survivors show long-term lethal effects of ionising radiations, which become apparent as precocious senescence $[1,4,27,28]$. Smith and Whitney [39] using the same HDF strain as in our experiments have demonstrated that even two sister cells arising from a single mitosis can differ in their ability to proliferate by as many as 8 PDs. In order to characterize possible conditions (if any) by which a "memory" of irradiation [27] is maintained in clonogenic survivors, it would become necessary to establish the interclonal and intraclonal variation in proliferative potentials from clonogenic survivors and control cells by frequency-distribution curves [39]. This means an extensive experimental task, which can only be performed by large laboratories.

\section{CONCLUSIONS}

This study to test the influence of a single acute irradiation on the replicative potential of confluent HDF cultures in phase II with either X-rays or fast neutrons has shown that the apparent precocious senescence is wholly attributable to the loss of potentially clonogenic cells (see case (1) of Discussion). There was no indication of radiation late effects as in the form of reduced replicative potentials of clonogenic survivors by 
which the irradiated cultures were repopulated. This finding is in keeping with the view emerging from in vivo studies $[22,30,31]$ that ionising radiations do not accelerate the ageing process.

\section{ACKNOWLEDGEMENTS}

This work was financed by the Fraunhofer-Gesellschaft e.V., Munich, F.R.G., and was carried out with technical assistance from Mrs. Ch. Kunze in the Laboratorium für Experimentelle Radiologie, D-8042 Neuherberg. Neutron radiation facilities were kindly provided by Drs. F. Schulz and B. Hietel of the G.S.F., Neuherberg. The authors are indebted to Professor Dr. O. Messerschmidt for his abiding support and encouragement.

\section{REFERENCES}

1 A. Macieira-Coelho, C. Diatloff and E. Malaise, Doubling potential of fibroblasts from different species after ionising radiation. Nature, 261 (1976) 586.

2 A. Macieira-Coelho, C. Diatloff and E. Malaise, Concept of fibroblast aging in vitro: Implications for cell biology. Gerontology, 23 (1977) 290.

3 M. Namba, K. Nishitani and T. Kimoto, Carcinogenesis in tissue culture. 29: Neoplastic transformation of a normal diploid cell strain, WI-38 with Co-60-gamma rays. Jpn. J. Exp. Med., 48 (1978) 303

4 A. Macieira-Coelho, C. Diatloff, C. Billardon, C. A. Bourgeois and E. Malaise, Effect of low dose rate ionising radiation on the division potential of cells in vitro. III. Human lung fibroblasts. Exp. Cell Res., 104 (1977) 215.

5 A. Weismann, Aufsätze über Vererbung und verwandte biologische Fragen, Fischer, Jena, 1892.

6 T. Cremer, August Weismann's contribution to cytogerontology. In Conference on Structural Pathology and Biology of Ageing, Zentrallaboratorium für Mutagenitätsprüfung, Jahreskonferenz 1979, Deutsche Forschungsgemeinschaft ed., Bold Verlag, 1980, pp. 283-306.

7 A. Carrel, On the permanent life of tissues outside of the organism. J. Exp. Med., 15 (1912) 516.

8 A. H. Ebeling, The permanent life of connective tissue outside of the organism. J. Exp. Med., 17 (1913) 273.

9 L. Hayflick and P. S. Moorhead, The serial cultivation of human diploid cell strains. Exp. Cell Res., 25 (1961) 585.

10 L. Hayflick, The limited in vitro lifetime of human diploid cell strains. Exp. Cell Res., 37 (1965) 614.

11 T. B. L. Kirkwood and R. Holliday, Commitment to senescence: a model for the finite and infinite growth of diploid and transformed human fibroblasts in culture. J. Theor. Biol, 53 (1975) 481 .

12 R. Holliday, FASEB Conference: Growth and death of diploid and transformed human fibroblasts. Fed. Proc., 34 (1975) 51.

13 R. Holliday, L. I. Huschtscha, G. M. Tarrant and T. B. L. Kirkwood, Testing the commitment theory of cellular aging. Science, 198 (1977) 366.

14 L. E. Orgel, The maintenance of the accuracy of protein synthesis and its relevance to aging. Proc. Natl. Acad. Sci. U.S.A., 49 (1963) 517.

15 L. E. Orgel, Aging of clones of mammalian cells. Nature, 243 (1973) 441.

16 D. L. Wilson, The programmed theory of aging. In M. Rockstein, M. L. Sussman and J. Chesky (eds.), Theoretical Aspects of Aging, Academic Press, New York, 1974, pp. 11-21.

17 L. Hayflick, The cellular basis for biological aging. In C. E. Finch and L. Hayflick (eds.), Handbook of the Biology of Aging, van Nostrand/Reinhold, New York, 1977, pp. 159-186. 
18 G. M. Martin, Cellular aging-Clonal senescence (A review-Part I). Am. J. Pathol., 89 (1977) 484.

19 T. B. L. Kirkwood, Evolution of aging (Review Article). Nature, 270 (1977) 301.

20 E. L. Schneider, H. Sternberg, R. Tice, G. C. Senula, D. Kram, J. R. Smith and G. Bynum, Cellular replication and aging. Mech. Ageing Dev., 9 (1979) 313.

21 S. Russ and G. M. Scott, Biological effects of gamma irradiation. Br. J. Radiol., 12 (1939) 440.

22 H. E. Walburg, Radiation-induced life shortening and premature aging. Adv. Radiat. Biol., 5 (1975) $145-179$.

23 O. Hug and A. M. Kellerer, Stochastik der Strahlenwirkung, Springer Verlag, Berlin, 1966.

24 L. Lima, E. Malaise and A. Macieira-Coelho, Effect of low dose-rate irradiation on the division potential of chick embryonic fibroblasts. Exp. Cell Res., 73 (1972) 345.

25 A. Macieira-Coelho, C. Diatloff and E. Malaise, Effect of low dose-rate irradiation on the division potential of cells in vitro. II. Mouse lung fibroblasts. Exp. Cell Res., 100 (1976) 228.

26 A. Macieira-Coelho, C. Diatloff, M. Billard, B. Fertil, E. Malaise and D. Fries, Effect of low doserate irradiation on the division potential of cells in vitro. IV. Embryonic and adult human lung fibroblast-like cells. J. Cell. Physiol., 95 (1978) 235.

27 G. Laublin, P. J. Deschavanne and E. P. Malaise, Effect of ionizing radiations on the lifespan of non-transformed human fibroblasts. Int. J. Radiat. Biol., 36 (1979) 281.

28 S. Ban, O, Nikaido, T. Ikushima and T. Sugahara, Doubling life of cultured human diploid cells after single exposure of X-rays and thermal neutrons. Radiat. Res., 81 (1980) 120.

29 S. Fukushima, Effect of X-irradiation on the clonal lifespan and fission rate in Paramecium aurelia. Exp. Cell Res., 84 (1974) 267.

30 J. R. Maisin, A. Decleve, G. B. Gerber, G. Mattelin and M. Lambiet-Collier, Chemical protection against the long-term effects of a single whole-body exposure of mice to ionising radiation. II. Causes of death. Radiat. Res., 74 (1978) 415.

31 S. C. Finch and G. W. Beebe, Review of thirty years study of Hiroshima and Nagasaki atomic bomb survivors. II. Biological effects on aging. J. Radiat. Res. Suppl., 16 (1975) 108.

32 E. L. Schneider and E. J. Stanbridge, A simple biochemical technique for the detection of $M y c o-$ plasma contamination of cultured cells. Methods Cell Biol., 10 (1975) 277-290.

33 A. F. G. Stevenson, T. Cremer and O. Messerschmidt, Erholung und zy togerontologische Aspekte der menschlichen Bindegewebszellen nach Röntgen- und Neutronenbestrahlung. In $O$. Messerschmidt, G. Möhrle and R. Zimmer (eds.), Strahlenschutz in Forschung und Praxis, Band XVIII, Georg Thieme Verlag, Stuttgart, 1977, pp. 20-29.

34 R. Cox and W. K. Masson, Changes in the radiosensitivity during the in vitro growth of diploid human fibroblasts. Int. J. Radiat. Biol., 26 (1974) 193.

35 V. J. Cristofalo and B. B. Sharf, Cellular senescence and DNA synthesis. Exp. Cell Res., 76 (1973) 419.

36 W. W. Nicholas, D. G. Murphy, V. J. Cristofalo, L. H. Toji, A. E. Greene and S. A. Dwight, Characterisation of a new human diploid cell strain, IMR-90. Science, 196 (1977) 60.

37 K. Kaji and M. Matsuo, Aging of chick embryo fibroblasts in vitro-I. Saturation density and population doubling rate. Exp. Gerontol., 13 (1978) 439.

38 H. Hoehn, E. M. Bryant and G. M. Martin, The replicative lifespans of euploid hybrids derived from short-lived and long-lived human skin fibroblast cultures. Cytogenet. Cell Genet., 21 (1978) 282.

39 J. R. Smith and R. G. Whitney, Intraclonal variation in proliferative potential of human diploid fibroblasts: stochastic mechanism for cellular aging. Science, 207 (1980) 82.

$40 \mathrm{~J}$. Z. Beer, Heritable lesions affecting proliferation of irradiated mammalian cells. Adv. Radiat. Biol., 8 (1979) 363-417.

41 M. Guichard, M. Tubiana and E. P. Malaise, Changes in repair of potentially lethal damage with culture age in EMT-6 cells. Int. J. Radiat. Biol., 35 (1979) 111.

$42 \mathrm{~J}$. R. Williams, Role of DNA repair in cell inactivation, aging and transformation: A selective review, a speculative model. Adv. Radiat. Biol., 6 (1976) 161-211.

43 L. E. Hopwood and L. J. Tolmach, Manifestations of damage from ionizing radiation in mammalian cells in the postirradiation generations. Adv. Radiat. Biol., 8 (1979) 317-362. 